\title{
On Numerical Simulation Approach for Multiple Resonance Modes in Servo Systems
}

\author{
Qixin Zhu, ${ }^{1,2}$ Hongli Liu, ${ }^{1}$ Yiyi Yin, ${ }^{3}$ Lei Xiong, ${ }^{2}$ and Yonghong $Z_{h u}{ }^{4}$ \\ ${ }^{1}$ School of Mechanical Engineering, Suzhou University of Science and Technology, Suzhou 215009, China \\ ${ }^{2}$ School of Electrical and Automation Engineering, East China Jiaotong University, Nanchang 330013, China \\ ${ }^{3}$ School of Electronic and Information Engineering, Suzhou University of Science and Technology, Suzhou 215009, China \\ ${ }^{4}$ School of Mechanical and Electronic Engineering, Jingdezhen Ceramic Institute, Jingdezhen 333001, China
}

Correspondence should be addressed to Qixin Zhu; bob21cn@163.com

Received 3 July 2017; Revised 21 October 2017; Accepted 12 November 2017; Published 29 November 2017

Academic Editor: Petko Petkov

Copyright (c) 2017 Qixin Zhu et al. This is an open access article distributed under the Creative Commons Attribution License, which permits unrestricted use, distribution, and reproduction in any medium, provided the original work is properly cited.

\begin{abstract}
Mechanical resonance is one of the most pervasive problems in servo control. Closed-loop simulations are requisite when the servo control system with high accuracy is designed. The mathematical model of resonance mode must be considered when the closed-loop simulations of servo systems are done. There will be a big difference between the simulation results and the real actualities of servo systems when the resonance mode is not considered in simulations. Firstly, the mathematical model of resonance mode is introduced in this paper. This model can be perceived as a product of a differentiation element and an oscillating element. Secondly, the second-order differentiation element is proposed to simulate the resonant part and the oscillating element is proposed to simulate the antiresonant part. Thirdly, the simulation approach for two resonance modes in servo systems is proposed. Similarly, this approach can be extended to the simulation of three or even more resonances in servo systems. Finally, two numerical simulation examples are given.
\end{abstract}

\section{Introduction}

Servo system is a feedback control system that is used to follow or reproduce a process accurately. One of the basic requirements in control systems is that they have the ability to regulate the controlled variables to reference commands without a steady-state error against unknown and unmeasurable disturbance inputs. Its main task is to achieve the power amplification, transformation, and control under the command requirements, so that the output torque, speed, and position control is very flexible and convenient. In many cases, the servo system means that output of the system is a feedback control system of mechanical displacement or displacement velocity and acceleration and its role is to enable the mechanical displacement output (or angular displacement) to accurately track the input (or angle). Its structures have no difference with other forms of feedback control systems in principle. The servo system is originally used for national defense, such as gun control, automatic driving ships, aircraft, and missiles. Later it gradually extended to many sectors of the national economy such as automatic machine, wireless tracking control, and semiconductor manufacturing equipment [1-6].

Mechanical resonance is one of the most common problems designers face when trying to maximize either command response or dynamic stiffness [7]. In the case of rotation, the drive and the drive shaft are generally considered rigid. In fact, the mechanical transmission shaft has different degrees of elastic torsion deformation. With the broadening of the frequency band of the system, the effect of the mechanical resonance caused by the elastic deformation on the dynamic characteristics of the system is obviously strengthened. From the transfer function of the system, it often contains a pair of conjugate zeroes and poles, which easily lead to mechanical resonance phenomenon. And this phenomenon is more obvious in the junction of feedback sensor and load connection in servo system [8].

Resonance is harmful in most cases. It causes mechanical deformation and greatly reduces the mechanical structure and even produces environmental pollution, and so on. In 


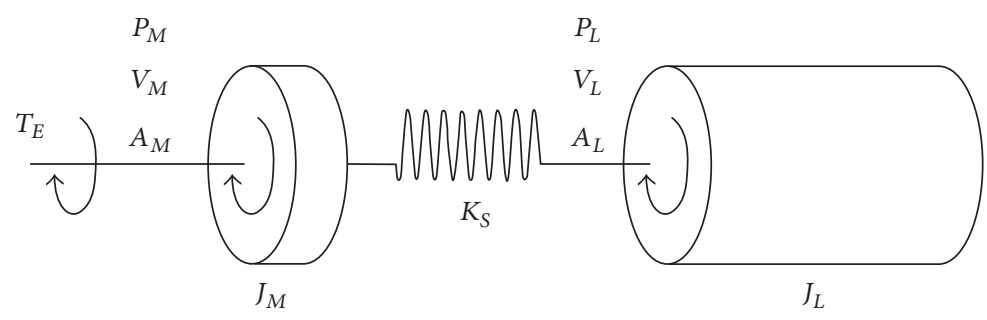

Figure 1: Diagram of compliant coupling between motor and load.

order to make the system have good dynamic performance, it is necessary to pay attention to control and eliminate resonance in servo control system. When resonance happened, the work of the excitation input system is balanced with the dissipated work phase, and the shape of the resonance peak is closely related to the damping. From the mechanical point of view, resonance precaution has the following measures: (1) improving the structure or the mechanical excitation to make the natural frequency avoid the excitation frequency; (2) adopting damping devices; (3) passing the resonance fast when there is a mechanical starting or stopping. From the control point, the low-pass or notch filter is often used to reduce the influence of resonance in the system [9-12]. Other theories and approaches such as resonance ratio control [13] and acceleration feedback [14] have been put forward to decrease the influence of resonance mode $[15,16]$. The simulation of closed-loop is requisite when analyzing the influence of the resonance modes in servo system. And the mathematical model of the resonance mode must be considered in the simulation; otherwise the closed-loop simulation results will differ from the actual servo system greatly. Therefore the study of numerical simulation of resonant modes has great significance. For the simulation of the resonance mode, only the simulation of single resonance mode is reported [17]. And the simulation of multiple resonance modes is still open. This motivates us to do this study.

Based on the analysis of the mathematical model of resonance mode, the resonance mode is perceived as a product of a second-order differentiation element and an oscillating element. And the second-order differentiation element is proposed to simulate the resonant part and the oscillating element is proposed to simulate the antiresonant part. The simulation approach for multiple resonance modes in servo systems is proposed in this paper.

\section{Mathematical Model of Resonance Mode}

Mechanical resonance is one of the most pervasive problems in motion control. Resonance is caused by springiness or compliance between two inertias. Most often, resonance is caused by compliance in transmission between motor and load. It is shown in Figure $1[10,15]$, where $T_{E}$ is electromagnetic torque, $P_{M}$ is motor position, $V_{M}$ is motor velocity, $A_{M}$ is motor acceleration, $P_{L}$ is load position, $V_{L}$ is load velocity, $A_{L}$ is motor acceleration, $J_{M}$ is the moment of inertia for motor, $J_{L}$ is the moment of inertia for load, $K_{C V}$ is velocity compliance coefficient, and $K_{S}$ is position compliance. Once

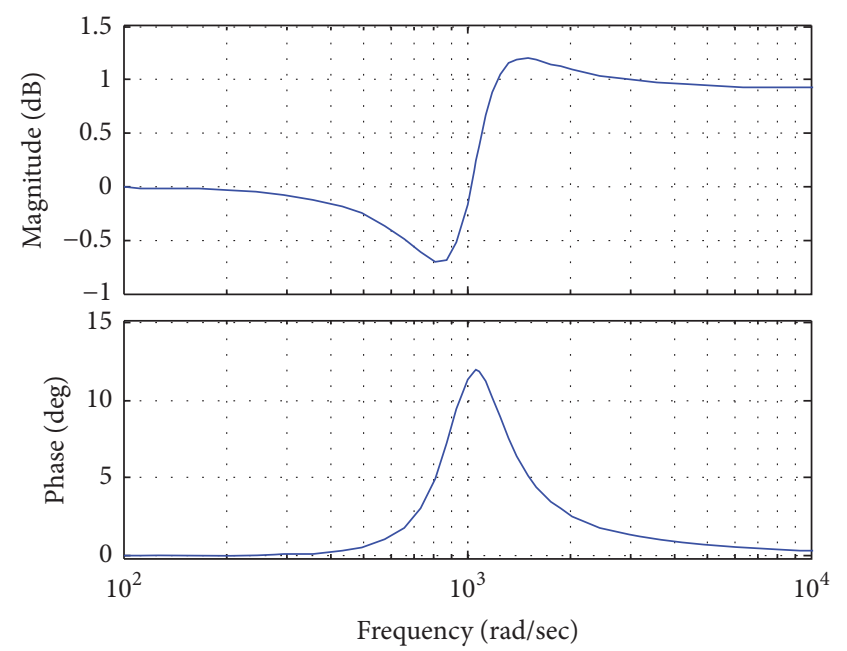

FIGURE 2: Bode diagram of resonance mode.

the motor shaft begins to turn, the combined transmission compliance winds up, which will produce torque to the load in proportion to the difference between motor and load positions.

When there is only one resonance mode in the system, the model of the resonance is expressed as follows $[9,10,18]$ :

$$
R(s)=\frac{J_{L} s^{2}+K_{C V} s+K_{S}}{\left(\frac{J_{M} J_{L}}{J_{M}+J_{L}}\right) s^{2}+K_{C V} s+K_{S}} .
$$

The Bode diagram of this model is shown in Figure 2.

\section{The Simulation of Single Resonance Mode}

It is very difficult to get the value of $K_{C}$ and $K_{C V}$ in (1) and users care little about these two values in applications. But the values of resonant frequency $w_{R}$, resonant amplitude $M_{\mathrm{R}}$, antiresonant frequency $w_{\mathrm{AR}}$, and antiresonant amplitude $M_{\mathrm{AR}}$ should be cared about. We can design the resonance mode shown in (1) if these four parameters are given. In the simulation of resonance mode, the following model can be considered:

$$
L(s)=\frac{w_{n 2}^{2}}{w_{n 1}^{2}} \frac{s^{2}+2 \xi_{1} w_{n 1} s+w_{n 1}^{2}}{s^{2}+2 \xi_{2} w_{n 2} s+w_{n 2}^{2}} .
$$




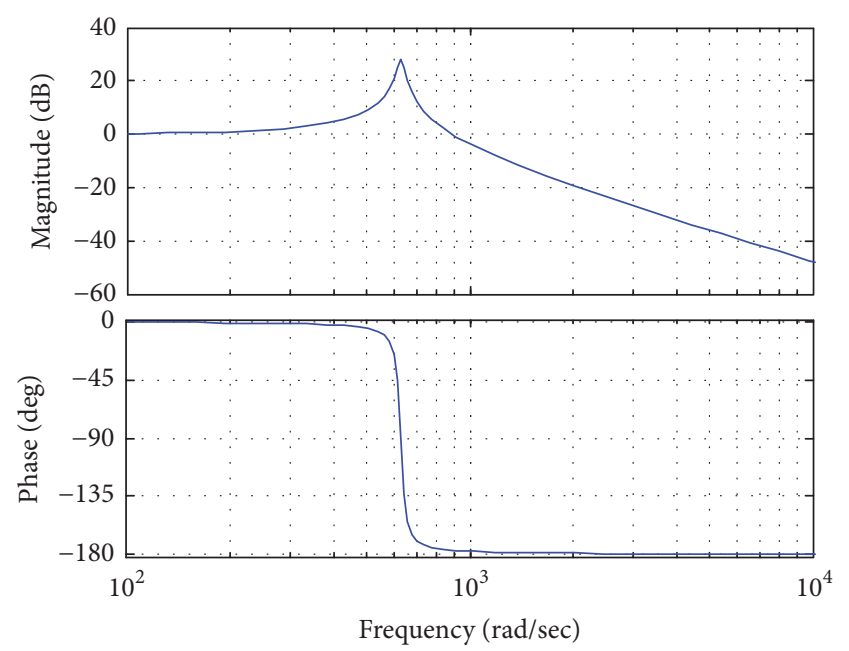

Figure 3: Bode diagram of typical oscillating element.

We now consider setting the values of $\xi_{1}, \xi_{2}, w_{n 1}$, and $w_{n 2}$ to make the frequency response of $L(s)$ similar to its expected frequency response.

This model is a product of a second-order differentiation element and an oscillating element. At low frequency, coefficient $w_{n 2}^{2} / w_{n 1}^{2}$ makes the magnitude of the model's frequency character turn to zero, which will not affect the low frequency character of the position loop object.

Before determining the undetermined coefficients in (2), we would consider the oscillating element

$$
\mathrm{ZD}(s)=\frac{w_{n 2}^{2}}{s^{2}+2 \xi_{2} w_{n 2} s+w_{n 2}^{2}} .
$$

Given $\xi_{2}=0.02$ and $w_{n 2}=200 \pi \mathrm{rad} / \mathrm{s}$, the frequency character of $\mathrm{ZD}(s)$ is shown in Figure 3. This Bode diagram shows a resonance clearly, and our purpose is to use the magnitude of this resonance $M_{2}$ to generate the resonant magnitude $M_{\mathrm{R}}$ in resonance model. It is easy to prove that the resonant frequency of this oscillating element occurs at $w_{r 2}$, and it follows that [19]

$$
\begin{aligned}
& M_{2}=\frac{1}{2 \xi_{2} \sqrt{1-\xi_{2}^{2}}} \quad\left(\xi_{2} \leq \frac{\sqrt{2}}{2}\right), \\
& w_{r 2}=w_{n 2} \sqrt{1-2 \xi_{2}^{2}} .
\end{aligned}
$$

If the resonant magnitude is known, we can use it to get damping by rewriting formula (4) into the following:

$$
\xi_{2}=\sqrt{\frac{1-\sqrt{1-M_{2}^{-2}}}{2}}, \quad\left(M_{2}<1, \quad \xi_{2}<\frac{\sqrt{2}}{2}\right) .
$$

But the resonant magnitude unit in (4) and (6) is not $\mathrm{dB}$, and if we want to use the known resonant magnitude $M_{\mathrm{R}}$ (unit is $\mathrm{dB}$ ) to calculate proper damping, the resonant magnitude

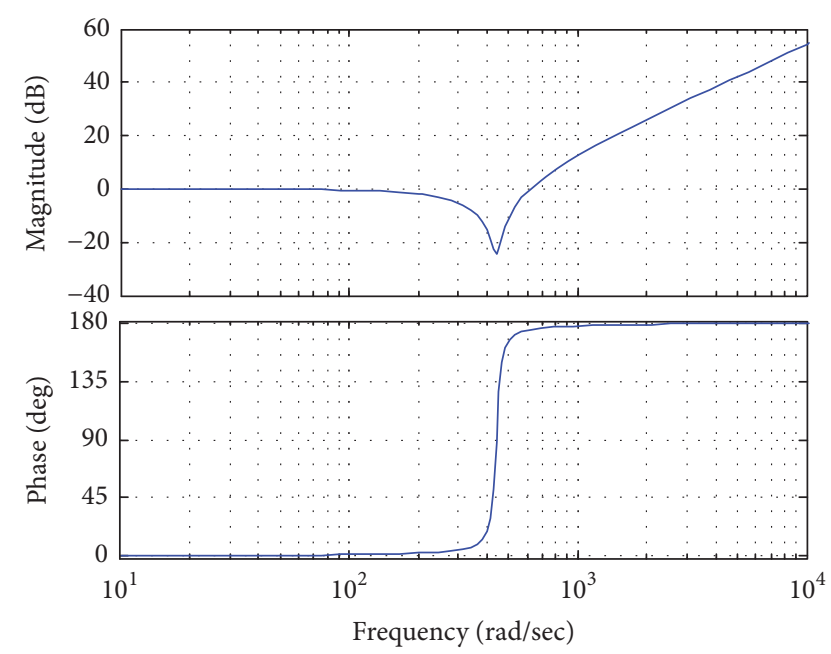

Figure 4: Bode diagram of typical differential model.

whose unit is $\mathrm{dB}$ should be changed into corresponding resonant magnitude whose unit is not $\mathrm{dB}$. The transformation formula is shown as follows:

$$
M_{2}=10^{M_{\mathrm{R}} / 20} \text {. }
$$

Substitute (7) into (6), to obtain

$$
\xi_{2}=\sqrt{\frac{1-\sqrt{1-10^{-M_{\mathrm{R}} / 10}}}{2}}, \quad\left(M_{\mathrm{R}}>0, \xi_{2}<\frac{\sqrt{2}}{2}\right) .
$$

When resonant magnitude of resonance mode is small, it has little influence in servo systems. The resonant magnitudes which we need to consider generally have large value. Since the damping $\xi_{2}$ is small in (8), we can argue through (5) that

$$
w_{r 2} \approx w_{n 2} \text {. }
$$

When simulating resonance modes, we can simply consider the resonant frequency as $w_{n 2}$. If the resonant frequency $w_{\mathrm{R}}$ and the resonant magnitude $M_{\mathrm{R}}$ are known, we can know $\xi_{2}$ and $w_{n 2}$; thus the model of oscillating element shown in (3) can be defined.

Then we consider the second-order differentiation element in (2).

$$
\mathrm{WF}(s)=\frac{s^{2}+2 \xi_{1} w_{n 1} s+w_{n 1}^{2}}{w_{n 1}^{2}}
$$

Given $\xi_{1}=0.03$ and $w_{n 1}=140 \pi \mathrm{rad} / \mathrm{s}$, the frequency character of $\mathrm{WF}(s)$ is shown in Figure 4.

The Bode diagram clearly shows an antiresonance, and our purpose is to use the magnitude of this resonance $M_{1}$ to generate the antiresonant magnitude $M_{\mathrm{AR}}$. It can be easily proved that the antiresonant frequency of the element occurs at $w_{r 1}$, and it follows that

$$
\begin{aligned}
\xi_{1} & =\sqrt{\frac{1-\sqrt{1-M_{1}^{2}}}{2}}, \quad\left(M_{2}<1, \xi_{1}<\frac{\sqrt{2}}{2}\right), \\
w_{r 1} & =w_{n 1} \sqrt{1-2 \xi_{1}^{2}} .
\end{aligned}
$$


But the unit of antiresonant magnitude in (11) is not $\mathrm{dB}$, and if antiresonant magnitude $M_{\mathrm{AR}}$ (unit $\mathrm{dB}$ ) is known and we use it to calculate proper damping, the antiresonant magnitude whose unit is $\mathrm{dB}$ should be changed into corresponding antiresonant magnitude whose unit is not $\mathrm{dB}$. The transformation formula is

$$
M_{1}=10^{M_{\mathrm{AR}} / 20} \text {. }
$$

Substitute (13) into (11), to obtain

$$
\xi_{1}=\sqrt{\frac{1-\sqrt{1-10^{M_{\mathrm{AR}} / 10}}}{2}}, \quad\left(M_{\mathrm{AR}}<0, \xi_{1}<\frac{\sqrt{2}}{2}\right) .
$$

Since the sign of the antiresonant magnitude $M_{\mathrm{AR}}$ is minus, the damping rate $\xi_{1}$ is small. We can argue through (12) that

$$
w_{r 1} \approx w_{n 1} \text {. }
$$

When simulating resonance modes, we can simply consider the antiresonant frequency as $w_{n 1}$. If antiresonant frequency $w_{\mathrm{AR}}$ and antiresonant magnitude $M_{\mathrm{AR}}$ are known, we can calculate $\xi_{1}$ and $w_{n 1}$; that is to say, the model of second-order differentiation element shown in (10) is defined.

Calculate (3) to obtain

$$
\begin{array}{r}
20 \log |\mathrm{ZD}(j w)|=-20 \log \sqrt{\left(1-\lambda_{2}^{2}\right)^{2}+4 \xi_{2}^{2} \lambda_{2}^{2}}, \\
\lambda_{2}=\frac{w}{w_{n 2}} .
\end{array}
$$

Calculate (10) to obtain

$$
\begin{aligned}
& 20 \log |\mathrm{WF}(j w)|=20 \log \sqrt{\left(1-\lambda_{1}^{2}\right)^{2}+4 \xi_{1}^{2} \lambda_{1}^{2}}, \\
& \lambda_{1}=\frac{w}{w_{n 1}} .
\end{aligned}
$$

Add formulas (16) and (17), and set $w=w_{n 1}$ and $w=w_{n 2}$; then $M_{\mathrm{AR}}, M_{\mathrm{R}}$ can be calculated as

$$
\begin{aligned}
& 20 \log \sqrt{\left(1-1^{2}\right)^{2}+4 \xi_{1}^{2} \times 1^{2}} \\
& -20 \log \sqrt{\left(1-\left(\frac{w_{n 1}}{w_{n 2}}\right)^{2}\right)^{2}+4 \xi_{2}^{2}\left(\frac{w_{n 1}}{w_{n 2}}\right)^{2}}=M_{\mathrm{AR}}, \\
& 20 \log \sqrt{\left(1-\left(\frac{w_{n 2}}{w_{n 1}}\right)^{2}\right)^{2}+4 \xi_{1}^{2}\left(\frac{w_{n 2}}{w_{n 1}}\right)^{2}} \\
& -20 \log \sqrt{\left(1-1^{2}\right)^{2}+4 \xi_{2}^{2} \times 1^{2}}=M_{\mathrm{R}} .
\end{aligned}
$$

Simplify formula (18) to obtain

$$
\begin{gathered}
4 \frac{\xi_{1}^{2}}{M_{1}^{2}}-4\left(\frac{w_{n 1}}{w_{n 2}}\right)^{2} \xi_{2}^{2}=\left(1-\left(\frac{w_{n 1}}{w_{n 2}}\right)^{2}\right)^{2}, \\
4\left(\frac{w_{n 2}}{w_{n 1}}\right)^{2} \xi_{1}^{2}-4 \xi_{2}^{2} M_{2}^{2}=-\left(1-\left(\frac{w_{n 2}}{w_{n 1}}\right)^{2}\right)^{2} .
\end{gathered}
$$

This is a system of binary quadric equations about $\xi_{1}^{2}, \xi_{2}^{2}$ and one can calculate $\xi_{1}, \xi_{2}$ through solving the equality system. When the values of $\xi_{1}, \xi_{2}, w_{n 1}$, and $w_{n 2}$ are gotten, the model of formula (2) can be gotten. That is to say, the mathematical model of a resonance mode is gotten.

\section{The Simulation of Multiple Resonance Modes}

If the system has two resonance modes, the main idea of the simulation is as follows:

(1) Setting up the mathematical model of these two resonance modes separately

(2) Multiplying these two mathematical models together

(3) Fine-tuning the values of damping ratio.

The flow chart for the simulation of two resonance modes is shown in Figure 5. What we need to explain is that two resonance modes have coupling effect at the resonant frequency and antiresonant frequency when the Bode diagrams of two resonance modes are superposed, so the given $M_{\mathrm{AR} 1}$ and $M_{\mathrm{R} 1}$ or designed $\xi_{11}$ and $\xi_{12}$ and the given $M_{\mathrm{AR} 2}$ and $M_{\mathrm{R} 2}$ or designed $\xi_{21}$ and $\xi_{22}$ will not meet the requirements. In this case, we could fine-tune the values of $\xi_{11}, \xi_{12}, \xi_{21}$, and $\xi_{22}$ by a way of circulation based on the sign of the difference of $M_{\mathrm{AR} 1}$ and $M_{\mathrm{AR} 2}$ or $M_{\mathrm{R} 1}$ and $M_{\mathrm{R} 2}$. The principle of fine tuning is that the smaller the damping ratio is, the larger the resonance magnitude is and the larger the damping ratio is, the smaller the resonance magnitude is.

Similarly, this approach can be extended to the simulation of three or even more resonances in servo systems.

\section{Numerical Simulation Examples}

Example 1. Generate one resonance mode by the approach in this paper. The resonance mode has the antiresonant frequency of $200 \mathrm{~Hz}$ and the antiresonant magnitude of $20 \mathrm{~dB}$, while the resonant frequency is $300 \mathrm{~Hz}$ and the resonant magnitude is $20 \mathrm{~dB}$. Using the approach in this paper, the numerical model of this resonance mode can be gotten by

$$
L(s)=\frac{3.553 \times 10^{6} s^{2}+2.499 \times 10^{8} s+5.611 \times 10^{12}}{1.579 \times 10^{6} s^{2}+3.755 \times 10^{8} s+5.611 \times 10^{12}} .
$$

The Bode diagram of this resonance mode is shown in Figure 6. We can see clearly an antiresonance which has a magnitude of $-20 \mathrm{~dB}$ and a resonance which has a magnitude of $20 \mathrm{~dB}$ that meet the requests.

Example 2. Generate two resonance modes by the approach in this paper. One resonance mode has the antiresonant frequency of $220 \mathrm{~Hz}$ and the antiresonant magnitude of $20 \mathrm{~dB}$, while the resonant frequency is $310 \mathrm{~Hz}$ and the resonant magnitude is $20 \mathrm{~dB}$. Another resonance mode has the antiresonant frequency of $540 \mathrm{~Hz}$ and the antiresonant 


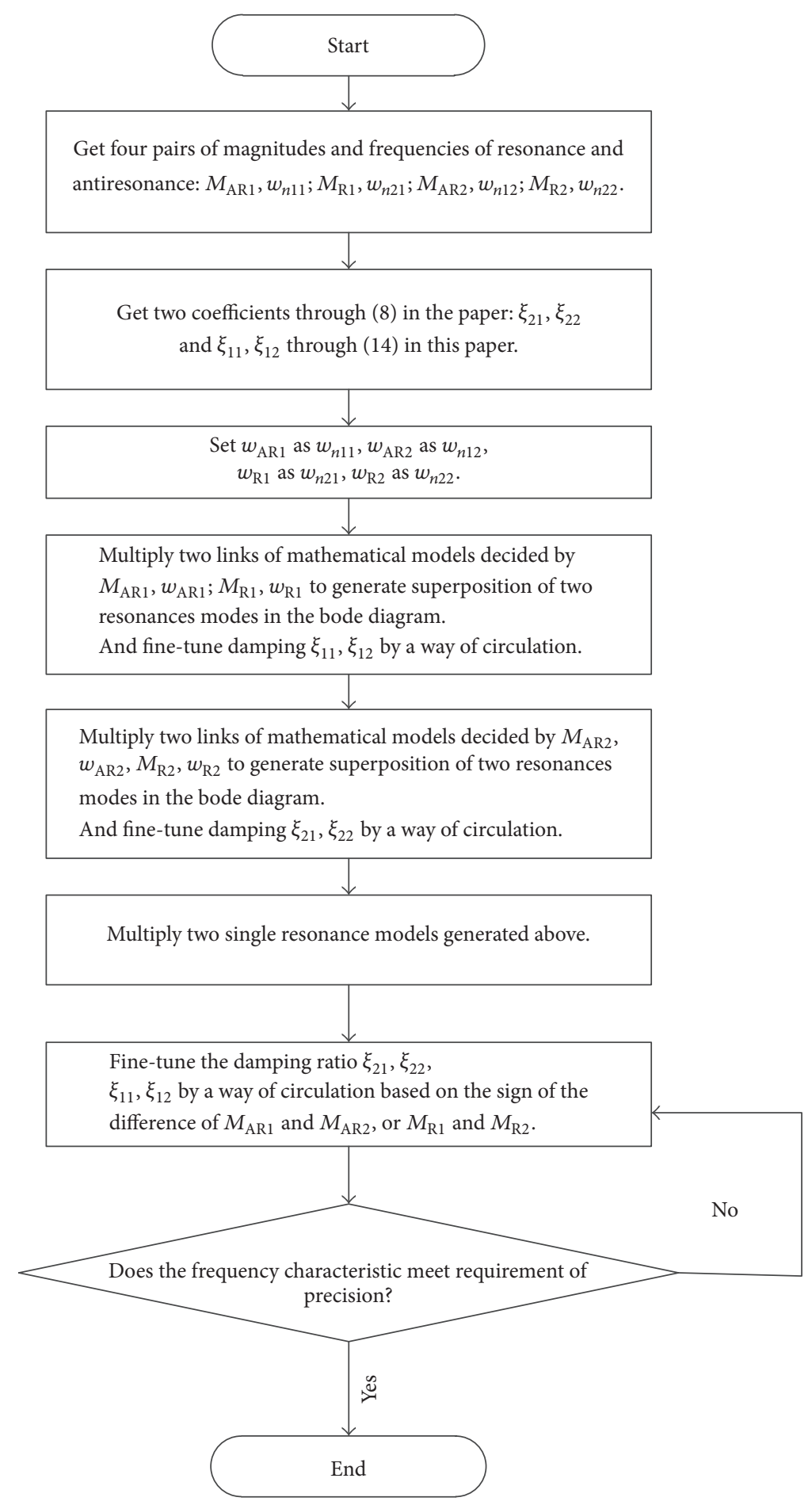

FIGURE 5: The flow chart for numerical simulation of two resonance modes.

magnitude of $10 \mathrm{~dB}$, while the resonant frequency is $690 \mathrm{~Hz}$ and the resonant magnitude is $15 \mathrm{~dB}$. Using the approach in this paper, the numerical model of this resonance mode can be gotten by

$$
L(s)=\frac{7.131 \times 10^{13} s^{4}+1.884 \times 10^{16} s^{3}+9.582 \times 10^{20} s^{2}+8.715 \times 10^{22} s+1.569 \times 10^{27}}{2.20 \times 10^{13} s^{4}+2.783 \times 10^{16} s^{3}+5.01 \times 10^{20} s^{2}+1.615 \times 10^{23} s+1.569 \times 10^{27}} .
$$




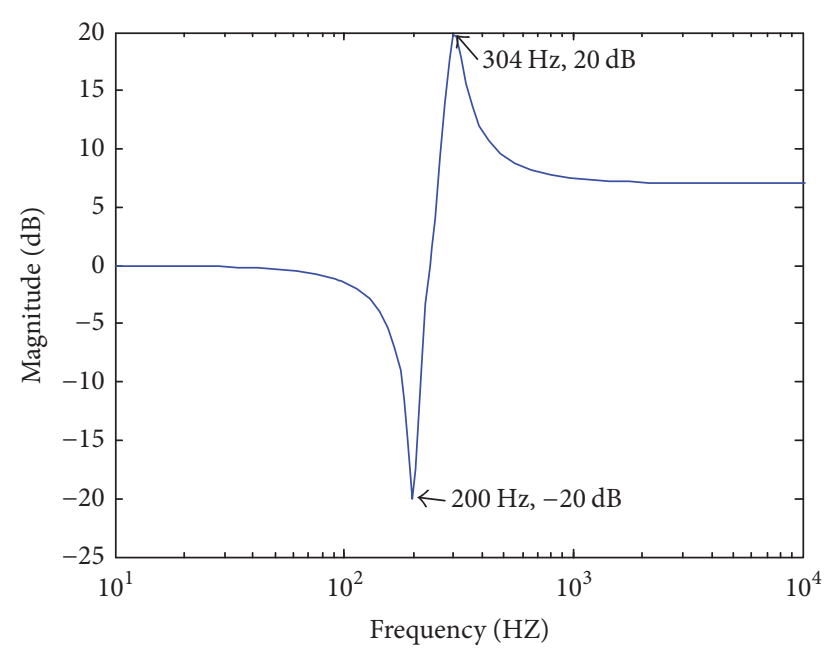

FIGURE 6: Bode diagram of one resonance mode.

The Bode diagrams of the modes are shown in Figure 7. We can see clearly from the Bode diagrams that there is an antiresonance which has a magnitude of $-19.9 \mathrm{~dB}$ at the frequency of $220 \mathrm{~Hz}$, a resonance which has a magnitude of $19.4 \mathrm{~dB}$ at the frequency of $309 \mathrm{~Hz}$, an antiresonance of $-10 \mathrm{~dB}$ at the frequency of $540 \mathrm{~Hz}$, and a resonance magnitude of $15 \mathrm{~dB}$ at $695 \mathrm{~Hz}$ that meet the requests.

\section{Conclusions}

The mathematical model of resonance modes is introduced in this paper. The second-order differentiation element is proposed to simulate the resonant part and the oscillating element is proposed to simulate the antiresonant part; the numerical simulation of a single resonant mode is realized in this way. In the case of the simulation of multiple resonance modes, one needs to multiply the simulated single resonance modes and fine-tuning damping to meet accuracy requirements. When fine-tuning the damping ratio, one should use a method of circulation based on the sign of the difference of two resonant magnitudes. The principle of fine tuning is that the smaller the damping ratio is, the larger the resonant magnitude is and the larger the damping ratio is, the smaller the resonant magnitude is.

This approach of simulation can be applied to the research on eliminating or compensating the effects of mechanical resonance in servo system and also the optimization of servo system, and so on. An interesting topic for further study is the method of fine-tuning damping since the multiple resonances will have the coupling effect.

\section{Conflicts of Interest}

The authors declare that there are no conflicts of interest regarding the publication of this paper.

\section{Acknowledgments}

This work was partly supported by National Natural Science Foundation of China under Grants 51375323 and 61563022,

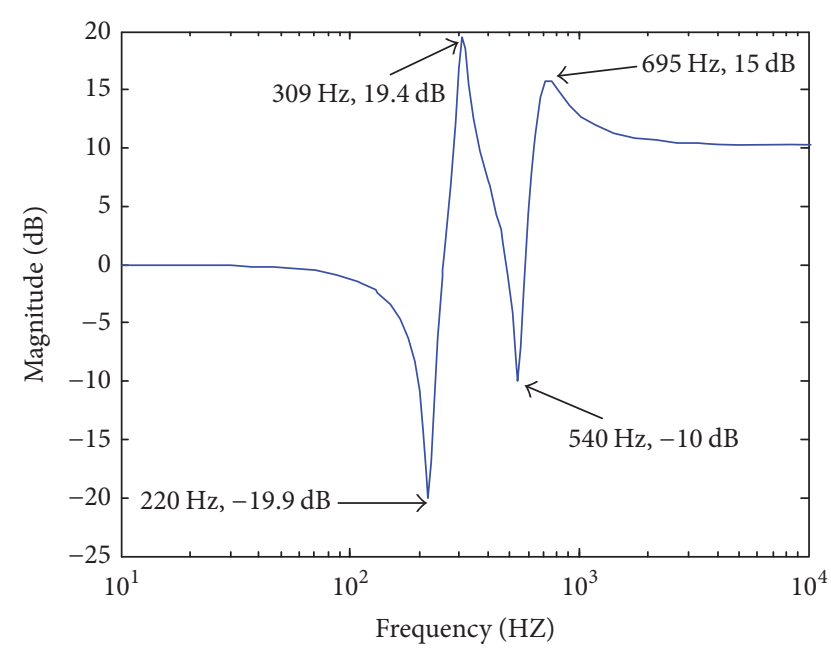

FIGURE 7: Bode diagrams of two resonance modes.

Major Program of Natural Science Foundation of Jiangxi Province, China, under Grant 20152ACB20009, Scientific Research Foundation of Suzhou University of Science and Technology under Grant XKZ201409, Foundation of Jiangsu Key Laboratory of Intelligent Building Energy Efficiency, and Qing Lan Project of Jiangsu Province, China.

\section{References}

[1] X. H. Jiao, J. Y. Zhang, and T. L. Shen, "An adaptive servo control strategy for automotive electronic throttle and experimental validation," IEEE Transactions on Industrial Electronics, vol. 61, no. 11, pp. 6275-6284, 2014.

[2] S. Hara, Y. Yamamoto, T. Omata, and M. Nakano, "Repetitive control system: a new type servo system for periodic exogenous signals," IEEE Transactions on Automatic Control, vol. 33, no. 7, pp. 659-668, 1988.

[3] S. H. Zhao, Y. S. Chen, and Y. L. Mao, "Adaptive load observerbased feed-forward control in PMSM drive system," Transactions of the Institute of Measurement and Control, vol. 37, no. 3, pp. 414-424, 2015.

[4] R. Delpoux and T. Floquet, "High-order sliding mode control for sensorless trajectory tracking of a PMSM," International Journal of Control, vol. 87, no. 10, pp. 2140-2155, 2014.

[5] M. Taktak-Meziou, A. Chemori, J. Ghommam, and N. Derbel, "Linear and nonlinear MPC for track following in the design of HDD servo systems," International Journal of Systems, Control and Communications, vol. 6, no. 1, pp. 20-47, 2014.

[6] Q. X. Zhu and L. Xiong, "Higher order modeling of position loop plant and controller design in PMSM servo system," Innovative Computing, Information and Control Express Letters, Part B: Applications, vol. 6, no. 6, pp. 1691-1696, 2015.

[7] G. Ellis and R. D. Lorenz, "Comparison of motion control loops for industrial applications," in Proceedings of the IEEE Industry Applications Conference, 34th IAS Annual Meeting, pp. 25992605, October 1999.

[8] Q. Hu and H. Tong, "Research of servo system mechanical resonance," Electric Drive, no. 3, pp. 7-11, 2000.

[9] Q. X. Zhu, H. L. Liu, and N. Krys, "A new design of notch filter in servo systems," Microcomputer Information, vol. 24, no. 7-1, pp. 56-58, 2008 (Chinese). 
[10] R. Cao, S. H. Wang, and J. B. Sun, "Speed control with vibration suppression of two-inertia servo system based on resonance ratio control," in Proceedings of the 17th International Conference on Electrical Machines and Systems (ICEMS '14), pp. 2227-2231, October 2014.

[11] J. Kang, S. L. Chen, and X. G. Di, "Online detection and suppression of mechanical resonance for servo system," in Proceedings of the 3rd International Conference on Intelligent Control and Information Processing (ICICIP '12), pp. 16-21, July 2012.

[12] W.-Y. Wang and A.-W. Shen, "Detection and reduction of middle-frequency resonance for industrial servo with selftuning lowpass filter," Journal of Control Science and Engineering, vol. 2012, Article ID 478907, 12 pages, 2012.

[13] M. Yang, H. Hu, and D. G. Xu, "Cause and suppression of mechanical resonance in PMSM servo system," Electric Machines and Control, vol. 16, no. 1, pp. 79-84, 2012 (Chinese).

[14] S. B. Wang, X. M. Ren, and G. F. Sun, "Suppression of mechanical resonance based on dynamic surface control and acceleration feedback," in Proceedings of the 33rd Chinese Control Conference (CCC '14), pp. 7927-7932, July 2014.

[15] G. Ellis and R. D. Lorenz, "Resonant load control methods for industrial servo drives," in Proceedings of the 35th IAS Annual Meeting and World Conference on Industrial Applications of Electrical Energy, pp. 1438-1445, October 2000.

[16] M. Wang, X. W. Liu, X. S. Dong, G. Xiong, Z. Shen, and R. Bai, "A novel method to detect and suppress mechanical resonance for servo system," in Proceedings of the 9th International Symposium on Computational Intelligence and Design (ISCID '16), pp. 349-354, December 2016.

[17] D. D. Ji, H. L. Liu, Q. X. Zhu et al., “The numerical simulation approach for resonance modes of servo mechanical system," Aerospace Control, vol. 34, no. 3, pp. 73-76, 94, 2016 (Chinese).

[18] G. Ellis, Control System Design Guide, Academic Press, Boston, Mass, USA, 2000.

[19] S. S. Hu, Automatic Control Theory, Science Press, Beijing, China, 2013 (Chinese). 


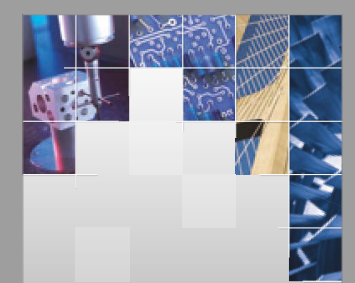

\section{Enfincering}
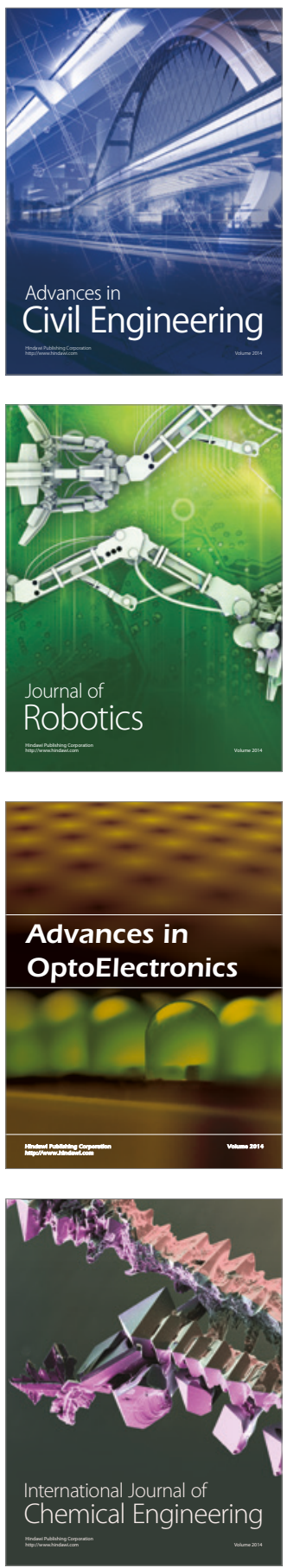

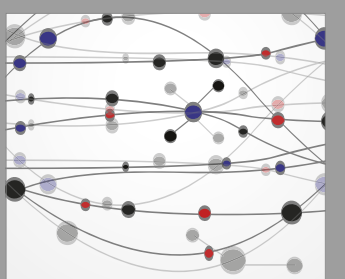

The Scientific World Journal

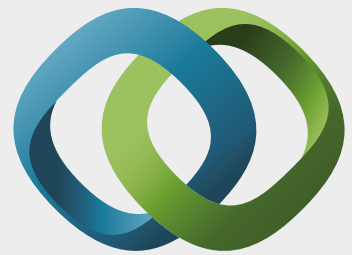

\section{Hindawi}

Submit your manuscripts at

https://www.hindawi.com
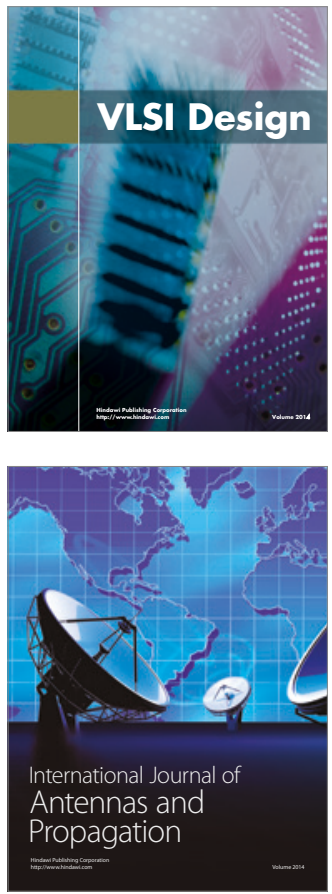

\section{Rotating}

Machinery
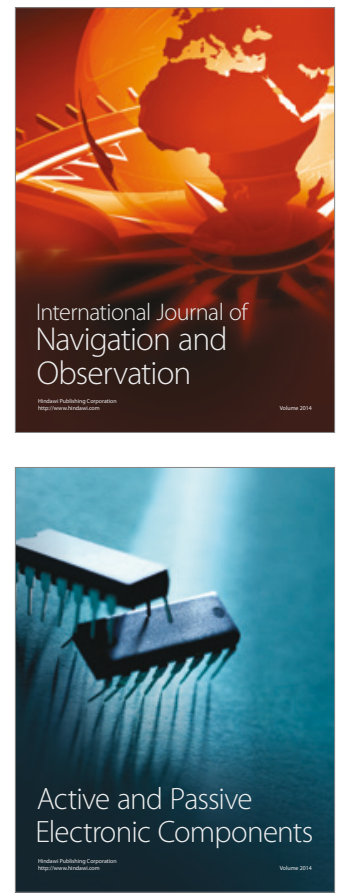
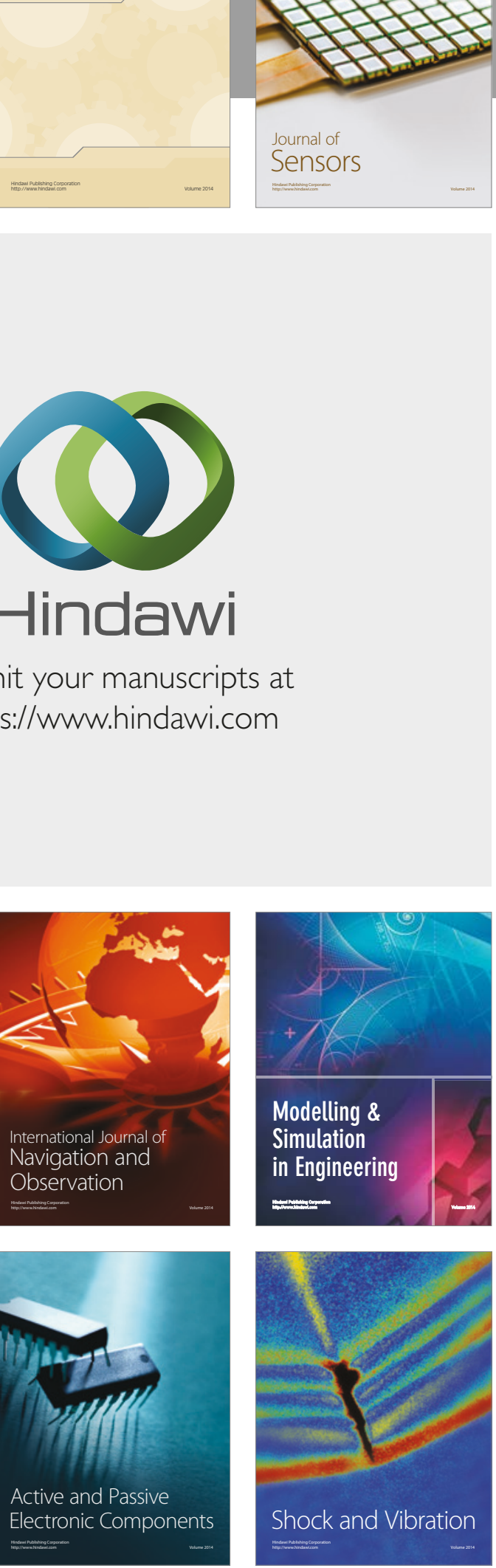
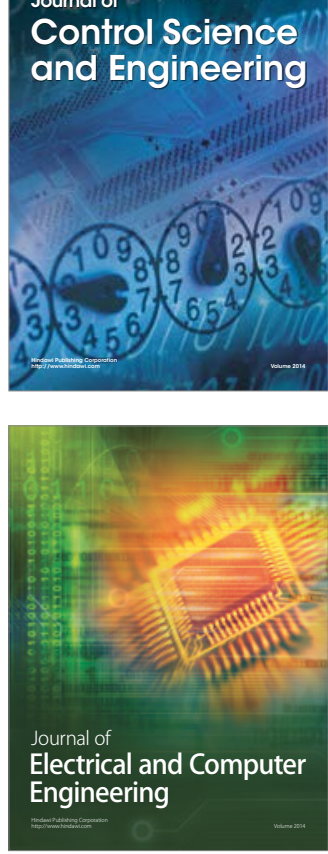

Distributed

Journal of

Control Science

and Engineering
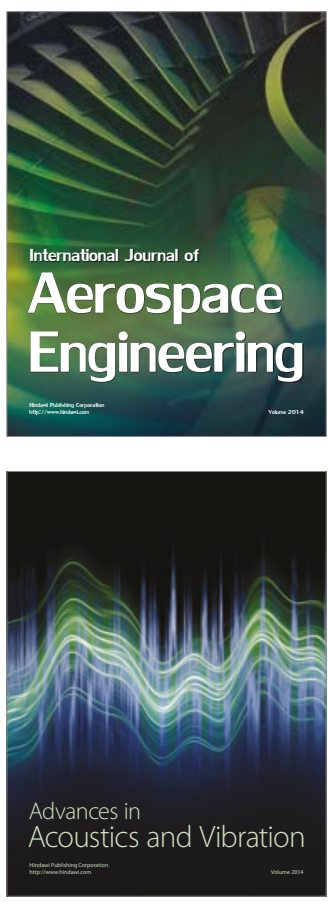

Sensor Networks 